\section{Breakout of Vaping-associ- ated Lung Injuries (VALI) 2019: A Public Health Threat}

\section{Sir,}

A previously healthy 20 years young man presented in the Emergency Department with worsening shortness of breath for the last two days. He denied any history of alcohol use or smoking. He also denied any history of using additives while vaping. There was no fever, no recent travel history or flu-like illness. He was a collegestudent with no occupational exposure to any allergens or industrial fumes. He gave the history of using flavoured e-cigarettes for 2-3 months. Later, he developed intractable cough and was prescribed oral azithromycin by his primary physician, with no relief. On physical examination, he was febrile, tachycardic and tachypneic with oxygen saturation of $66 \%$ on room air. He had bilateral crepitation on lung auscultation.

Laboratory work-up showed leukocytosis with neutrophilic shift (total white cell counts $20 \times 10^{9} / \mathrm{L}$ with neutrophils $18 \times 10^{9} / \mathrm{L}$ ). Septic work-up showed raised C-reactive protein of $30 \mathrm{mg} / \mathrm{L}$. Moreover, echocardiogram was unremarkable. He got intubated due to hypoxemic respiratory failure, increased work of breathing with respiratory rate of 50 breaths per minute. There were multiple tiny nodules with no definite infiltrates or consolidation seen on chest $\mathrm{X}$-ray (Figure 1 ). His blood gas values postintubation showed $\mathrm{pH}$ of $7.34, \mathrm{PCO}_{2}$ of $50 \mathrm{~mm} \mathrm{Hg}$ and $\mathrm{PO}_{2}$ of 75 $\mathrm{mm} \mathrm{Hg}$. Rest of the tests' results were unremarkable. Piperacillin-tazobactam was administered as intravenous antibiotics and he was shifted to the intensive care unit. Later, he got extubated, observed for two days in ward and was discharged uneventfully. CT chest was not performed due to financial restraints. With the background of vaping with flavoured eliquid, it was strongly suspected that his vaping exposure posed a diagnostic challenge. Other possibilities for differential diagnosis such as viral or bacterial pulmonary infections, occupational lung diseases or cardiac conditions were excluded. Such case made us to be knowledgeable about vaping-related lung injury (VALI).

Battery operated electronic cigarettes or e-cigarettes are used to inhale nicotine and additive agents. There has been no largescale study identifying such exposure till 2019, when several journals and health departments reporting such cases as a public health concern. Potentially toxic chemicals such as nicotine, carbonyls, benzene, toluene are shown as key ingredients invaping.

According to the Center of Disease Control (CDC), VALI is defined as the use of e-cigarette or vaping in the 90 days before symptom onset, pulmonary infiltrate (opacities on chest X-ray or ground glass opacities on computed tomography), absence of infection after workup and no evidence of an alternative cause (cardiac, rheumatologic, or neoplastic). ${ }^{1-4}$

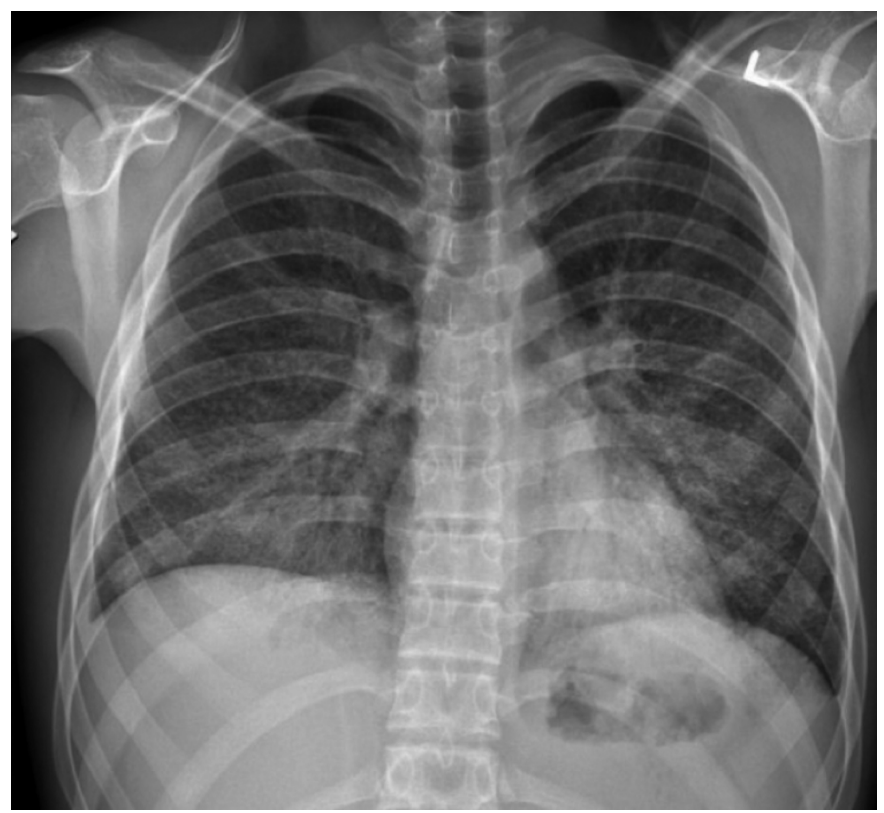

Figure 1: ChestX-ray showing bilateral nodularity in lung fields.

Vaping devices in the form of easy-to-carry pens and USB flash drives have been aggressively marketed. It was first introduced in the US in 2006. Literature highlighted the short and long term health effects of vaping and its comparison with traditional cigarettes. CDC reported VALI with worsening symptoms including shortness of breath, fever, cough, vomiting, diarrhea, headache, dizziness and chest pain. Range of symptoms and imaging were correlated with chemical pneumonitis. Patients required ICU care with mechanical ventilation due to severe respiratory failure.

As of early September 2019, approximately 450 cases in the United States were reported with mortality in five cases. ${ }^{5,6}$ The largest case number (150-199) was reported from Texas and Ilinois of United States with 47 deaths. ${ }^{2}$

The pathogenesis of VALI is yet to be discovered; however, multiple compounds were identified as potential risk factors such as diacetyl, nickel, tin, and lead; no specific liquid or device has been identified as the causative agent. VALI has been reported with nicotine, tetrahydrocannabinol and cannabidiol products. Laboratory and imaging findings are non-specific. Bronchoscopy samples showed neutrophilia and lipid laden macrophages.

The patterns of lung injury reported with VALI include acute eosinophilic pneumonia, lipoid pneumonia, acute lung injury and acute respiratory distress syndrome, acute and subacute hypersensitivity pneumonitis, organizing pneumonia, acute eosinophilic pneumonia, diffuse alveolar hemorrhage, and respiratory bronchiolitis-associated pneumonitis.,

Emergency physicians should inquire specifically about e-cigarette use in any patient presenting with acute lung disease. 
VALI is changing into an epidemic with under-reported numbers. In anticipation of rise of respiratory illnesses during influenza season, the CDC updated its guidance for VALI to help differentiate these patients from those with seasonal illnesses, like influenza. The guidance includes recommendations for physicians to ask patients with respiratory or gastrointestinal illness, if they have used vaping products or corticosteroids. Also, a reminder was included that not all patients with VALI need to be hospitalised; but they can be managed as outpatients if they have normal blood oxygen levels, no respiratory distress, and reliable access to health care. ${ }^{4-6}$ Regional data is non-existent till now. There is also a dire need to notify the reported cases to health authorities and spread the awareness about vaping, which may be considered as a safe option for smoking alternatives, its marketing and testing of e-liquid components.

\section{CONFLICT OF INTEREST:}

Authors declared no conflict of interest.

\section{AUTHORS' CONTRIBUTION:}

SS, MAB: Contributed in draft preparation and editing.

\section{REFERENCES}

1. CDC clinical outreach and communication activity. http://www.cdc.gov/. September 21, 2019.

2. Health and smoking and tobacco use; Electronic cigarettes. Center for Disease Control and prevention. http://www. cdc.gov/. September 21, 2019.
3. Ghinai I, Pray IW, Navon L, Laughlin OK, Saathoff HL, Hoots $\mathrm{B}$, et al. E-cigarette product use, or vaping, among persons with associated lung injury Illinois and Wisconsin, AprilSeptember 2019. MMWR Morb Mortal Wkly Rep 2019; 68(39):865-69.

4. Stanbrook MB. Vaping-associated lung illnesses highlight risks to all users of electronic cigarettes. CMAJ 2019; 191(48):E1319-E1320. doi: 10.1503/cmaj.191503.

5. Landman ST. Life-threatening bronchiolitis related to electronic cigarette use in a Canadian youth. CMAJ 2019; 191(48):E1321-E1331. doi: 10.1503/cmaj.191402.

6. Layden JE, Ghinai I, Pray I. Pulmonary illness related to ecigarette use in illinois and wisconsin preliminary report. $N$ Engl J Med 2020; 382:903-16.

Sadaf Sheikh ${ }^{1}$ and Muhammad Akbar Baig ${ }^{2}$

${ }^{1}$ Department of Emergency Medicine, Sultan Qaboos University Hospital, Muscat, Oman

${ }^{2}$ Department of Emergency Medicine, The Aga Khan University Hospital, Karachi, Pakistan

Correspondence to: Dr. Sadaf Sheikh, Sultan Qaboos University Hospital, Muscat, Oman

E-mail: sheikh.sadaf@gmail.com

Received: October 12, 2019; Revised: February 23, 2020;

Accepted: March 10, 2020

DOI: https://doi.org/10.29271/jcpsp.2020.11.1238 